Wahlsten, D., and Bishop, K.M. Effect sizes and meta-analysis indicate no sex differences in the corpus callosum. Behavioral and Brain Sciences, 1998, 21, 338-339.

Made available courtesy of Cambridge University Press: http://www.cambridge.org/

***Reprinted with permission. No further reproduction is authorized without written permission from the Cambridge University Press.

This version of the document is not the version of record.

Figures and/or pictures may be missing from this format of the document.***

\title{
Effect sizes and meta-analysis indicate no sex dimorphism in the human or rodent corpus callosum
}

\author{
Douglas Wahlsten and Katherine M. Bishop \\ Department of Psychology, University of Alberta, Edmonton, Alberta T6G \\ 2E9, Canada.wahlsten@psych.ualberta.ca
}

\begin{abstract}
Sexdimorphism occurs when group means differ by four or more standard deviations. H owever, the average size of the corpus callosum is greater in males by about one standard deviation in rats, 0.2 standard deviation in humans, and virtually zero in mice. F urthermore, variations in corpus callosum size are related to brain size and are not sex specific.
\end{abstract}

Fitch and $D$ enenberg $(F \& D)$ present evidence that ovarian hormones play an active role in rat brain development. We agree and will instead focus on four matters where we differ.

First, F\&D and many others (e.g., Constant \& Ruther 1996) refer to statistically significant differences as evidence of sex "dimorphism." This word is misleading. The Oxford English Reference Dictionary (Pearsall \& Trumble 1996) defines dimorphic as "exhibiting, or occurring in, two distinct forms" (p. 399), just as it defines dichotomy as "a division into two, esp. a sharply defined one" (p. 395). Examples of genuine sex dimorphism abound in nature; examples are the plumage of birds and the genitalia of mammals. The corpus callosum (CC), on the other hand, is not at all dimorphic in either rats or humans.

E ffect size compares the difference between group means to the standard deviation (S) within a group. F or sample data, $d=\left(M_{1}-\right.$ $M_{2}$ )/S estimates the population effect size $\delta$. Cohen (1992) regards $d$ values of $0.2,0.5$, and 0.8 as small, medium, and large, respectively, in published psychological research with humans. When $d$ is 1.0 , the difference between groups accounts for only $20 \%$ of the total variance, and scores of males and females overlap considerably. The degree of overlap can be found with Guttman's (1988) discrimination coefficient disco, which indicates the probability of identifying group membership from an individual's score.

Genuine dichotomy or dimorphism occurs when d is 4.0 or greater, or disco is close to 1.0 (F ig. 1). H owever, sex differences in the rat $C C$ in papers cited by $F \& D$ range from d of 0.85 to 1.35 . We have found $d$ is 1.25 for $C C$ area of Sprague-D awley rats. These are large effects but not at all dichotomous; many females have a larger CC than many males. Promulgating such group differences as a "dimorphism" grossly exaggerates the size of the sex difference. We propose that more accurate descriptors, especially effect size or disco, be employed. 


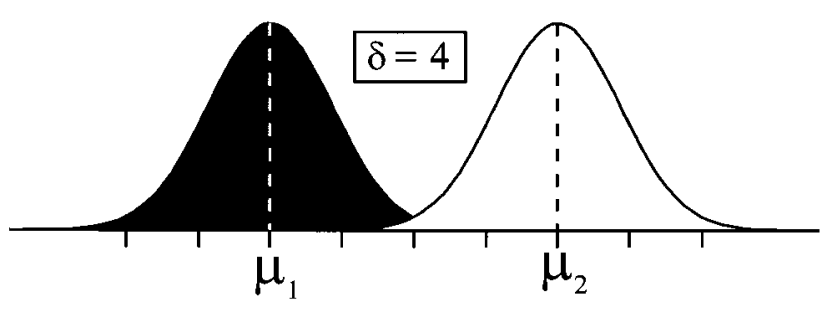

Figure 1 (Wahlsten $\&$ Bishop). F requency distributions of two populations whose means differ by four standard deviations.

Second, a superior estimate of the magnitude of a sex difference may be obtained by combining evidence from several independent studies with meta-analysis. Bishop and Wahlsten (1997) found the following $95 \%$ confidence intervals for the sex difference $\delta$ in 49 studies of humans: brain weight, 0.95-1.46 (larger in males); CC area, 0.13-0.29; ratio of area of splenium (posterior fifth, not anterior fifth as stated by $F \& D$ in sect. 6.2) to whole CC, $-0.25-0.02$. Thus, males had substantially larger brains on average and slightly larger CC area but no difference in CC shape.

When another study appears, its results should be combined with all previous studies in an updated, cumulative meta-analysis. For example, adding the study by Constant and Ruther (1996) changes the estimate of $\delta$ for CC area from 0.2092 based on 42 studies to 0.2048 and narrows the $95 \%$ confidence interval slightly (0.13-0.28). Once the literature becomes sufficiently voluminous, any new report could not nudge the estimate of $\delta$ a noteworthy amount, and the case can be closed. This we believe is well justified for sex differences in the human CC. H opefully, the onslaught of individually excellent but cumulatively uninformative research on this topic will soon cease.

Third, $F \& D$ argue that in general $C C$ size should not be corrected for whole brain size unless the correlation with brain size is large and significant. We disagree. By the allometric growth principle, we expect to find a larger CC in an individual with a larger brain, regardless of sex. Whether a sex difference in C C area is sex specific depends on whether the difference between male and female means exceeds what is expected from allometry. For example, with our data on 44 Sprague-D awley rats, the regression equation for predicting $\mathrm{CC}$ area from brain weight $(\mathrm{Y}=-0.89+$ $1.94 \mathrm{X}$ ) accounts for an $\mathrm{R}^{2}$ of 0.53 of variance in the CC. Adding sex to the equation increases $R^{2}$ nonsignificantly to 0.54 ; hence, there is no sex-specific effect. Fortunately, applying the regression method to adjust for brain size will not change the results for CC size if no relation exists. Thus, it is both safe and wise to make the adjustment. In the rat studies cited by $F \& D$, the $C C$ versus brain correlation is often nonsignificant because of small samples and low power. $M$ eta-analysis reveals a significant correlation of $r$ of 0.38 for six values from those studies in Table 2 that provide adequate details.

We join $F \& D$ in condemning the ratio method. A ratio is justified only when the relation is isometric (straight line through the origin, $Y$-intercept 0 ). CC versus brain size is an allometric relation and the $C C$ /brain ratio changes as a function of brain size, which means a ratio will not remove the influence of brain size from the data. In this case, the misuse of a ratio may create the appearance of a nonexistent effect or may mask a real effect. These artifactual mistakes will not be made with the regression method.

$F$ inally, $F \& D$ suggest that data from rats are relevant for the human brain. H owever, mice often show no sex difference in the anterior hypothalamus (the so-called sexually "dimorphic" nucleus) or in the CC. I f results with rats cannot be generalized to the genetically and ecologically closely related house mice, how can they be cited in support of arguments about the human brain? 